\title{
Novel somatic mutations and distinct molecular signature in aldosterone-producing adenomas
}

\author{
Tobias Åkerström 1 , Holger Sven Willenberg ${ }^{2}$, Kenko Cupisti ${ }^{3}$, Julian Ip ${ }^{4}$, \\ Samuel Backman', Ana Moser ${ }^{5}$, Rajani Maharjan', Bruce Robinson ${ }^{4}$, K Alexander Iwen ${ }^{5}$, \\ Henning Dralle ${ }^{6}$, Cristina D Volpe ${ }^{7}$, Martin Bäckdahl ${ }^{7}$, Johan Botling ${ }^{8}$, Peter Stålberg ${ }^{1}$, \\ Gunnar Westin', Martin K Walz ${ }^{9}$, Hendrik Lehnert ${ }^{5}$, Stan Sidhu ${ }^{4}$, Jan Zedenius ${ }^{7}$, \\ Peyman Björklund ${ }^{1}$ and Per Hellman ${ }^{1}$ \\ 'Department of Surgical Sciences, Uppsala University, Uppsala, Sweden \\ ${ }^{2}$ Department of Endocrinology and Metabolism, Rostock University Medical Center, Germany \\ ${ }^{3}$ General, Visceral and Pediatric Surgery University Hospital Düsseldorf, Düsseldorf, Germany \\ ${ }^{4}$ University of Sydney, Endocrine Surgical Unit and Cancer Genetics, Hormones and Cancer Group, Kolling Institute \\ of Medical Research, Royal North Shore Hospital, Sydney, Australia \\ ${ }^{5}$ Department of Medicine I, University of Lübeck, University Hospital, Lübeck, Germany \\ ${ }^{6}$ Department of General, Visceral and Vascular Surgery, University Hospital, University of Halle-Wittenberg, \\ Halle/Saale, Germany \\ ${ }^{7}$ Department of Molecular Medicine and Surgery, Endocrine Surgery Unit, Karolinska Institutet, Karolinska \\ University Hospital, Stockholm, Sweden \\ ${ }^{8}$ Department of Immunology, Genetics and Pathology, Uppsala University, Uppsala, Sweden \\ ${ }^{9}$ Klinik für Chirurgie und Zentrum für Minimal Invasive Chirurgie, Kliniken Essen-Mitte, Essen, Germany
}

Correspondence should be addressed to T Åkerström Email tobias.akerstrom@ surgsci.uu.se

\begin{abstract}
Aldosterone-producing adenomas (APAs) are found in 1.5-3.0\% of hypertensive patients in primary care and can be cured by surgery. Elucidation of genetic events may improve our understanding of these tumors and ultimately improve patient care. Approximately $40 \%$ of APAs harbor a missense mutation in the KCNJ5 gene. More recently, somatic mutations in CACNA1D, ATP1A1 and ATP2B3, also important for membrane potential/intracellular $\mathrm{Ca}^{2+}$ regulation, were observed in APAs. In this study, we analyzed 165 APAs for mutations in selected regions of these genes. We then correlated mutational findings with clinical and molecular phenotype using transcriptome analysis, immunohistochemistry and semiquantitative PCR. Somatic mutations in CACNA1D in 3.0\% (one novel mutation), ATP1A1 in $6.1 \%$ (six novel mutations) and $A T P 2 B 3$ in $3.0 \%$ (two novel mutations) were detected. All observed mutations were located in previously described hotspot regions. Patients with tumors harboring mutations in CACNA1D, ATP1A1 and ATP2B3 were operated at an older age, were more often male and had tumors that were smaller than those in patients with KCNJ5 mutated tumors. Microarray transcriptome analysis segregated KCNJ5 mutated tumors from ATP1A1/ATP2B3 mutated tumors and those without mutation. We observed significant transcription upregulation of CYP11B2, as well as the previously described glomerulosa-specific gene NPNT, in ATP1A1/ATP2B3 mutated tumors compared to KCNJ5 mutated tumors. In summary, we describe novel somatic mutations in proteins regulating the membrane potential/intracellular $\mathrm{Ca}^{2+}$ levels, and also a distinct mRNA and clinical signature, dependent on genetic alteration.
\end{abstract}

Key Words

- ATP1A1

- CACNA1D

- KCNJ5

- primary aldosteronism

- aldosterone-producing adenoma http://erc.endocrinology-journals.org DOI: $10.1530 /$ ERC-15-0321
(C) 2015 Society for Endocrinology Printed in Great Britain
Published by Bioscientifica Ltd
Endocrine-Related Cancer

(2015) 22, 735-744 


\section{Introduction}

Primary aldosteronism (PA) is the most common endocrine cause of secondary hypertension with an estimated prevalence of $\sim 5-10 \%$ in unreferred hypertensive patients and up to $20 \%$ of those with grade III hypertension (Rossi et al. 2006, Fogari et al. 2007, Hannemann \& Wallaschofski 2012). Aldosterone-producing adenomas (APAs) represent a surgically curable form of PA and occur in $\sim 30 \%$ of PA patients (Rossi et al. 2006, Fogari et al. 2007). The aldosterone overproduction leads to increased $\mathrm{Na}^{+}$and $\mathrm{H}_{2} \mathrm{O}$ retention by the kidney, resulting in hypertension. Importantly, the excess aldosterone also promotes cardiac remodeling and vascular collagen deposition, augmenting cardiovascular disease in these patients when compared to patients with essential hypertension (Brilla \& Weber 1992, Rossi et al. 1997, Savard et al. 2013).

In the normal zona glomerulosa (ZG) cell, the plasma membrane is kept at a highly hyperpolarized state (Quinn et al. 1987). This hyperpolarization is mainly attributed to a dominant conductance of potassium ions over the cell membrane (Spät \& Hunyady 2004). Depolarizing the membrane potential increases intracellular $\mathrm{Ca}^{2+}$ and subsequently increases aldosterone production and secretion (Spät \& Hunyady 2004).

Choi et al. (2011) identified mutations in the KCNJ5 gene in both sporadic APAs and in a family with bilateral adrenal hyperplasia. Mutations affecting the selectivity filter of GIRK4 (encoded by the KCNJ5 gene) resulted in the loss of selectivity for potassium, increased sodium influx and subsequent membrane depolarization. The membrane depolarization raises intracellular calcium concentrations, leading to increased CYP11B2 transcription and subsequent aldosterone production (Oki et al. 2012). Multiple screening studies of over 1000 sporadic APAs have established a prevalence of $\sim 40 \%$ mutations in KCNJ5 in Western countries (Akerstrom et al. 2012, Boulkroun et al. 2012, Lenzini et al. 2015) and even higher in Asian countries (Taguchi et al. 2012, Kitamoto et al. 2015, Wang et al. 2015, Zheng et al. 2015).

More recently, somatic mutations in genes encoding an additional ion channel (CACNA1D) and two ATPases (ATP1A1 and ATP2B3) were discovered in APAs (Azizan et al. 2013, Beuschlein et al. 2013, Scholl et al. 2013). Also, germline mutations in CACNA1D and CACNA1H were described in patients with early onset PA (Scholl et al. 2013, Scholl et al. 2015). Similar to GIRK4, the protein products of these genes are ultimately involved in regulating the membrane potential and $\mathrm{Ca}^{2+}$ homeostasis in adrenal glomerulosa cells. To provide better knowledge of prerequisites for potential diagnostic and therapeutic targets, we aimed to investigate the frequency of mutations in CACNA1D, ATP1A1 and ATP2B3 in 165 APAs, and explore clinical and molecular phenotype.

\section{Materials and methods}

\section{Included patients and clinical annotation}

Tumor specimens were collected from 165 patients (65 males and 100 females) with diagnosed APA from six different tertiary referral centers. The diagnosis was established by raised aldosterone/renin ratio, positive confirmatory tests, lateralization studies (AVS and/or CT/MRI) and histopathological examination, according to routine protocols at the individual centers. Patients gave written informed consent and approvals from the local ethical committees were obtained.

Cortisol-producing adenomas $(n=4)$ and non-secreting adenomas $(n=4)$ were included for CYP11B2 expression analysis. Patients with cortisol-producing adenomas had elevated cortisol in serum and failure to suppress cortisol with dexamethasone and postoperative cure. Non-hormone secreting adenomas were detected incidentally during abdominal CT scan. These were deemed functionally silent after negative hormone analyses and were removed on suspicion of malignancy (size or other radiological signs).

\section{Molecular genetic analyses}

DNA/RNA was extracted using Allprep DNA/RNA mini kit, (Qiagen), AllPrep DNA/RNA FFPE Kit (Qiagen) or DNeasy Blood and tissue kit, (Qiagen). cDNA was prepared using Revertaid H-minus First strand cDNA synthesis Kit, (Fermentas, Waltham, MA, USA). In total, 90 (54.5\%) KCNJ5 mutations had previously been observed (Akerstrom et al. 2012). Hotspot regions in CACNA1D exons $8 \mathrm{~A}, 8 \mathrm{~B}, 16$ and $33, A T P 1 A 1$ exons 4,8 and 21 and $A T P 2 B 3$ exon 8 were analyzed in all tumors. In addition, tumors without KCNJ5 mutation $(n=75)$ were analyzed for fragments corresponding to regions in CACNA1D exons 5, 6, 13, 14, 23, 27, 32, 35, 36, 37, 38, 39, 40. When available, confirmatory PCR was performed on DNA from peripheral blood/surrounding normal tissue as well as in cDNA. Details on PCR conditions and primer sequences are available upon request. Sanger sequencing was conducted on amplified fragments (Beckman Coulter Genomics, Takeley, UK). Chromatograms were analyzed

Published by Bioscientifica Ltd. 
using CodonCode aligner Software 4.2.4 (CodonCode Corporation, Dedham, MA, USA). NCBI Reference Sequences; NP_001122312.1 and NP_000711 (exon 8B) for CACNA1D, NP_001153705.1for ATP1A1 and NP_001001344.1 for ATP2B3 were used for sequence alignment. Evolutionary conservation was determined using the ClustalW2 algorithm (Larkin et al. 2007).

Transcriptome analysis was performed on 12 APAs (six KCNJ5 mutated, three ATP1A1/2B3 mutated and three tumors without known mutation) using the SurePrint G3 Human Exon $4 \times 180 \mathrm{~K}$ Microarray (Agilent Technologies, Santa Clara, CA, USA). Data analysis was conducted using the GeneSpring Software v.13 (Agilent Technologies). Quality control of the included samples was performed in the GeneSpring v.13 Software using a flag-based method. Baseline transformation to the median of all samples was performed. Data were log-transformed and normalized using the quantile method. Unsupervised hierarchal clustering using $10 \%$ of probes with the highest variation in expression was performed in R. Using all probes, unsupervised hierarchal clustering based on Euclidean distances and complete linkage was obtained. Heat maps of the top 100 up- and downregulated transcripts in ATP1A1/2B3 mutated tumors compared to $K C N J 5$ mutated tumors were generated and $t$-tests were performed. Subsequently, the Benjamini-Hochberg method was used for multiple testing correction, and significantly differentially expressed transcripts were identified by volcano plots using fold change $>2$ together with a corrected $P$-value of $<0.05$.

The expression of CYP11B2 was measured using a custom assay (ThermoFisher Scientific, Waltham, MA, USA), with mRNA specific primers as previously described (Fallo et al. 2012). An mRNA specific GAPDH assay was used as an internal control (ThermoFisher Scientific). The relative CYP11B2 expression was compared between KCNJ5 mutated APAs $(n=14)$, ATP1A1 $(n=3)$ ATP2B3 $(n=2)$ using a calculated $2^{(-\Delta \Delta C T)}$ value (Livak \& Schmittgen 2001). Non-hormone-secreting adenomas $(n=4)$ and cortisol-producing adenomas $(n=4)$ were used as controls.
NPNT immunohistochemistry was performed on $6 \mu \mathrm{m}$ frozen sections from three ATP2B3 mutated tumors, one ATP1A1 mutated, 15 KCNJ5 mutated tumors, one section of normal kidney tissue and one section of normal adrenal tissue next to a non-hormone-secreting adenoma. Briefly, sections were deparaffinized in xylene and rehydrated through graded ethanol. Endogenous peroxidases were inhibited by incubation in hydrogen peroxide solution. Avidin/Biotin blocking (Vector Laboratories, Burlingame, CA, USA) was applied then followed by normal goat serum. The sections were incubated with an antibody against NPNT, 1:150 (HPA003711, Sigma-Aldrich, St Louis, MI, USA) for $2 \mathrm{~h}$ at room temperature. For detection, a biotinylated and HRP-conjugated goat anti-rabbit secondary antibody was used, 1:200 (Vector Laboratories). The sections were developed with DAB (Vector Laboratories) and counterstained with hematoxilin.

\section{Statistics}

The overall group effect was analyzed using Oneway ANOVA for parametric data and a Kruskal-Wallis test for categorical data. If significant group effects were present, the overall analysis was followed by post hoc comparisons between groups using Bonferroni-corrected MannWhitney's $U$-test or two-tailed $T$-test. Categorical data were analyzed by $\chi^{2}$-tests. Data are expressed with either mean \pm s.E.M. or median and range for non-parametric data. SPSS 22 (IBM) was used for statistical analysis.

\section{Results}

The proportion of mutations and corresponding phenotypes of the screened cohorts are summarized in Tables 1 and 2 respectively. Tumors from three of the centers contained more than 50\% KCNJ5 mutations (Table 1). These cohorts had relatively large tumors, with a mean size of $17.9 \mathrm{~mm} \pm 0.92$, and included many female patients (68\%), and most patients were hypokalemic (96\%). For the entire cohort, we observed a female gender

Table 1 Proportion of mutations in APAs stratified by cohorts

\begin{tabular}{|c|c|c|}
\hline Cohort & Number of APAs & WT \\
\hline Uppsala & 36 & $8(22.2 \%)$ \\
\hline Dusseldorf & 33 & $17(51.5 \%)$ \\
\hline Halle & 8 & $4(50.0 \%)$ \\
\hline Lübeck & 20 & $4(20.0 \%)$ \\
\hline Sydney & 34 & $15(44.1 \%)$ \\
\hline Stockholm & 34 & $7(20.6 \%)$ \\
\hline Summary & 165 & $55(33.3 \%)$ \\
\hline
\end{tabular}

\begin{tabular}{ccc}
\hline \multicolumn{1}{c}{$\boldsymbol{K C N J 5}$} & & $\boldsymbol{A T P 1 A 1}$ \\
\hline $25(69.4 \%)$ & & $1(2.8 \%)$ \\
$15(45.5 \%)$ & $1(3.0 \%)$ \\
$4(50.0 \%)$ & 0 \\
$13(65.0 \%)$ & $2(10.0 \%)$ \\
$14(41.2 \%)$ & $2(5.9 \%)$ \\
$19(55.9 \%)$ & $4(11.8 \%)$ \\
$90(54.5 \%)$ & $10(6.1 \%)$ \\
\hline
\end{tabular}

\begin{tabular}{ccc}
\hline ATP2B3 & CACNA1D \\
\hline $2(5.6 \%)$ & 0 \\
0 & 0 \\
0 & 0 \\
$1(5.0 \%)$ & 0 \\
0 & $3(8.8 \%)$ \\
$2(5.9 \%)$ & $2(5.9 \%)$ \\
$5(3.0 \%)$ & $5(3.0 \%)$ \\
\hline
\end{tabular}


overrepresentation for patients with KCNJ5 mutated tumors compared to patients without KCNJ5 mutated tumors ( $80 \%$ vs $38 \%, P<0.0001)$ (Table 2$)$. These patients were also operated at a younger age (44.9 years \pm 1.23 vs 53.1 years $\pm 1.34, P<0.0001)$. No difference in adenoma size $(19.4 \mathrm{~mm} \pm 0.80$ vs $16.5 \mathrm{~mm} \pm 1.27, P=0.17)$ or preoperative aldosterone levels was observed (1365 pmol/1 \pm 219 vs $1355 \mathrm{pmol} / \mathrm{l} \pm 145, P=0.97)$.

A total of $20(12.1 \%)$ mutations in CACNA1D, ATP1A1 and $A T P 2 B 3$ (Table 1) were identified. The somatic nature of the mutations was verified in all samples with available non-tumoral tissue (Supplementary Table 1, see section on supplementary data given at the end of this article). Mutations in CACNA1D were found in $3.0 \%$ of tumors. These mutations were located in exon 8A, 16 and 33, corresponding to segment 6 (S6) in repeats I and II, and S5 in repeat IV (Supplementary Fig. 1). We discovered a novel mutation in exon 8A (p.Val401Leu). Val401 is an evolutionary conserved amino acid located two positions upstream from the frequently mutated Gly403 residue (Supplementary Table 2). Somatic mutations in ATP1A1 were observed in 10/165 (6.1\%) and in ATP2B3 in 5/165 (3.0\%) APAs. The mutations in ATP1A1 comprised the previously described p.Leu104Arg (c.311T $>$ G) along with three novel deletions in the same area, as well as three novel deletions in the M9 domain (Supplementary Figs 2A and 3, and Supplementary Table 3). The mutations found in ATP2B3 corresponded to amino acid residues located in the M4 domain of the protein. These included two previously described deletions and two novel deletions (Supplementary Figs 2B and 4). All of the observed mutations were found in non-KCNJ5 mutated tumors.

We observed a significant difference in clinical phenotype in patients with $A T P 1 A 1 / 2 B 3$ and $C A C N A 1 D$ mutated tumors compared to patients with KCNJ5 mutated tumors (Table 2). ATP1A1/2B3 and CACNA1D mutated tumors were significantly smaller than KCNJ5 mutated APAs (11.2 $\mathrm{mm} \pm 0.94$ vs $19.4 \mathrm{~mm} \pm 0.80, P<0.0001)$. Patients were operated at an older age (53.7 years \pm 2.35 vs 44.9 years $\pm 1.23, P=0.008$ ), and they were more often male (65\% vs $20 \%, P<0.0005)$. No significant difference in preoperative aldosterone levels was observed (1671 pmol/l \pm 340 vs $1365 \mathrm{pmol} / 1 \pm 219, P=0.30$ ).

Transcriptome analysis and unsupervised hierarchal clustering using $10 \%$ of probes with the highest variation in expression segregated KCNJ5 mutated tumors (Fig. 1A). Likewise, principal component analysis on all probes segregated KCNJ5 mutated tumors (Fig. 2). Unsupervised hierarchal clustering on the complete set of probes segregated KCNJ5 mutated tumors, except for one

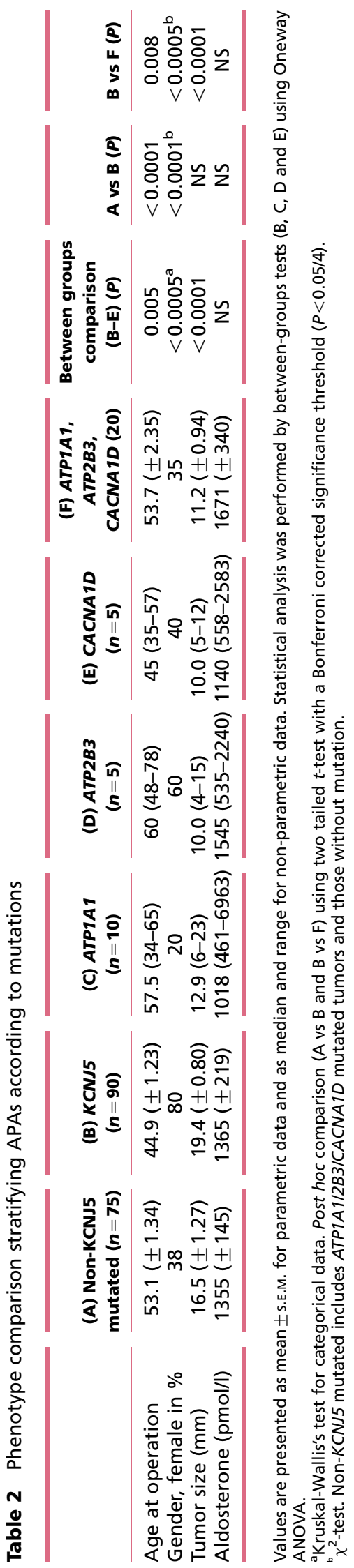

Published by Bioscientifica Ltd. 


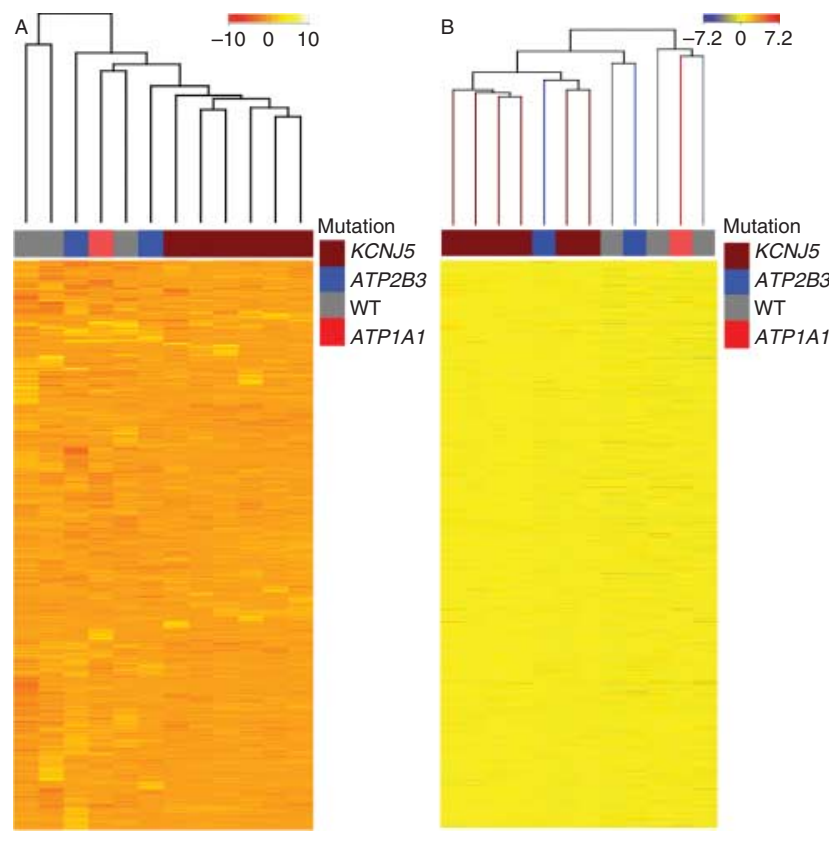

Figure 1

Transcriptional signal in APA samples. (A) Unsupervised hierarchal clustering based on Euclidean distances in 12 APAs (six KCNJ5 mutated, three $A T P 1 A 1 / 2 B 3$ mutated and three without known mutations $=\mathrm{WT}$ ) using $10 \%$ of probes with the highest variation in expression. (B) Heat map showing the results of unsupervised hierarchal clustering based on Euclidean distances and complete linkage of all quality passed probes in the microarray.

ATP2B3 mutant (Fig. 1B). The most up- and downregulated genes in $A T P 1 A 1 / 2 B 3$ mutated tumors compared to KCNJ5 mutated tumors are displayed in Supplementary Table 4 and Supplementary Fig. 5 (see section on supplementary data given at the end of this article). Only two transcripts, NPNT and RBM11, satisfied greater than twofold change with a false discovery rate (FDR) of $P<0.05$ (Fig. 3A). Comparing tumors without known mutation against KCNJ5 mutated tumors, we observed three genes with greater than twofold change and FDR $P<0.05$ (Fig. 3B). These included DNAH11 (FC change: -6.8 in KCNJ5 mutated, $P=0.013$ ), C1orf186 (FC change: +2.7 in KCNJ5 mutated, $P=0.013$ ) and PPP1R14A (FC change: +2.1 in KCNJ5 mutated, $P=0.048)$. No differences in the mRNA expression of ATP1A1, ATP2B3 or KCNJ5 were observed using the microarray. One probe for CYP11B2 was present on the microarray and showed higher expression in ATP1A1/2B3 mutated tumors than in KCNJ5 mutated (median signal: 0.99 vs -0.4$)$. To further investigate CYP11B2 expression, we conducted semiquantitative-PCR expression analysis in ATP1A1/2B3 and those with KCNJ5 mutation, confirming higher expression in $A T P 1 A 1 / 2 B 3$ mutated tumors $(P<0.05)$ (Fig. 4). To verify increased NPNT expression in $A T P 1 A 1 / 2 B 3$ tumors, we analyzed protein expression using immunohistochemistry. As expected, staining of normal kidney tissue revealed selective expression in glomeruli and blood vessels (Fig. 5A). In normal adrenal tissue, expression was restricted to ZG cells (Fig. 5B). Staining of tumor tissue confirmed array findings with higher expression of NPNT in ATP1A1/2B3 mutated APAs compared to KCNJ5 mutated APAs (Fig. 5C and D).

\section{Discussion}

In this study, we observed somatic mutations in $C A C N A 1 D$, $A T P 1 A 1$ and $A T P 2 B 3$ in $12.1 \%$ of APAs, including nine novel somatic mutations. CACNA1D mutations were detected in $3 \%$ of the tumors. CACNA1D encodes the alpha-1 subunit of an L-type voltage-dependent $\mathrm{Ca}^{2+}$ channel (VDCC) important for increasing intracellular $\mathrm{Ca}^{2+}$ and aldosterone production in ZG cells (Cohen et al. 1988). The observed mutations were located in segment 6 in repeats I and II, and S5 in repeat IV (Fig. 6). Interaction between S5 and S6 is important for the normal function of the channel pore (Catterall 2010). In vitro studies have shown that alterations in these portions of the protein lead to increased channel activity (Azizan et al. 2013, Scholl et al. 2013). We detected a novel missense mutation (p.Val401Leu) in one of the tumors. This position is located two amino acid residues upstream from the Gly403 in S6 (repeat I), one of the most frequently mutated residues in CACNA1D (Azizan et al. 2013, Scholl et al. 2013,

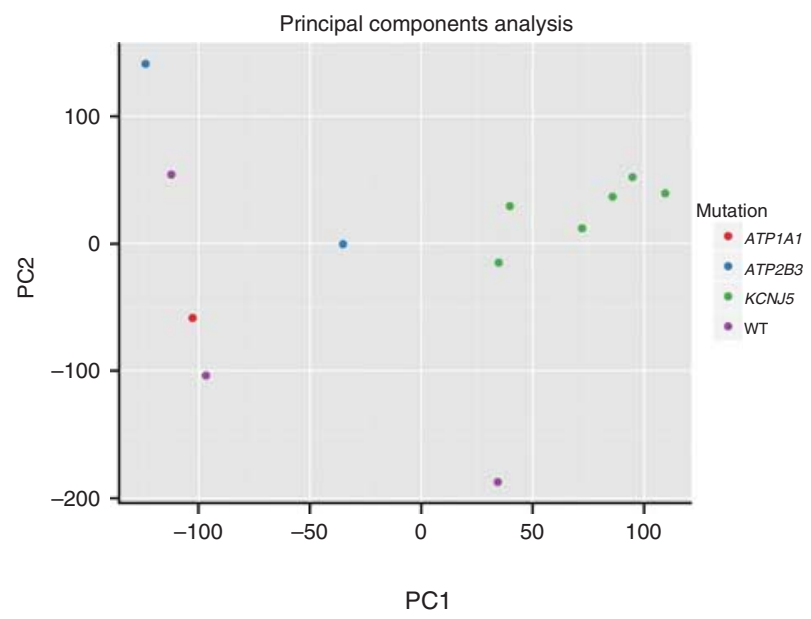

Figure 2

Principal component analysis on the transcriptional signals in APAs. Two-dimensional PCA on 12 APAs based on all probes. 

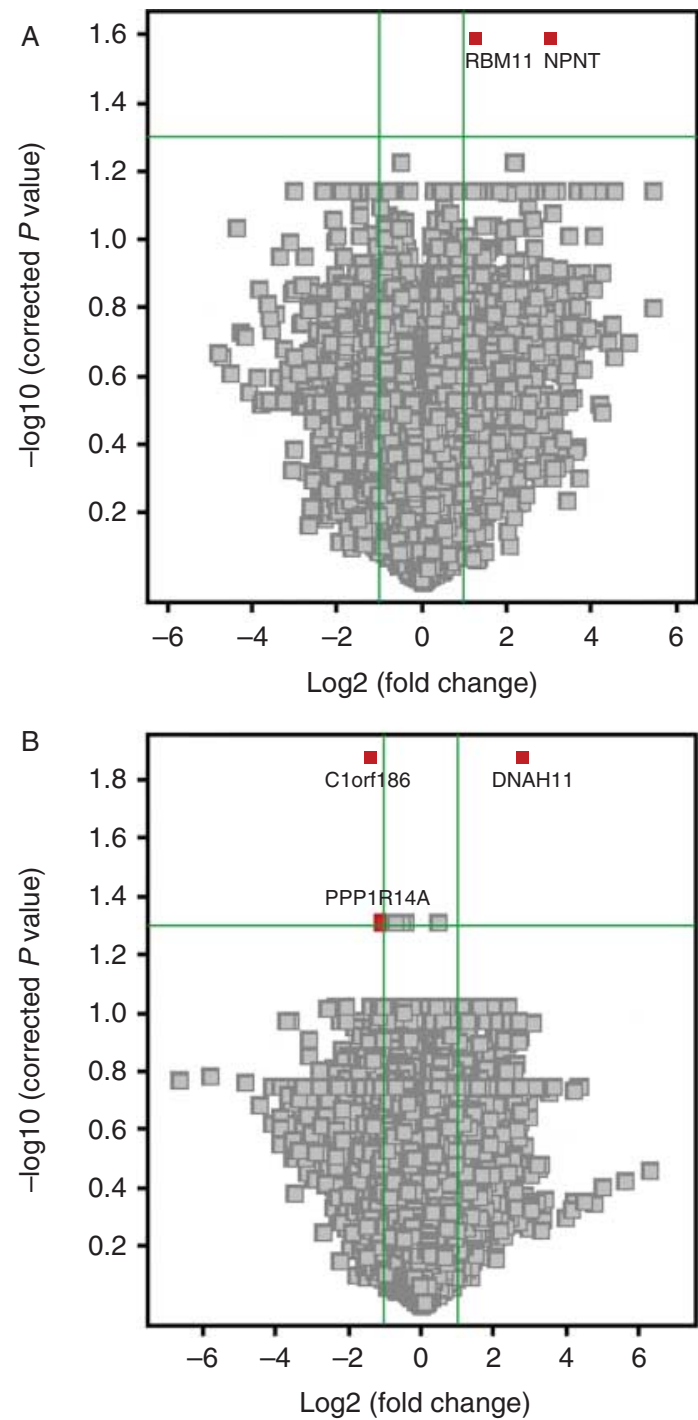

Figure 3

Statistically significant expression differences in ATP1A1/2B3/NT compared to KCNJ5 mutated APA. Volcano plot on all quality passed probes on the microarray. The $x$-axis shows the fold change $(\log 2)$ and the $y$-axis shows corrected $P$ value ( $-\log 10)$. $T$-tests were performed on greater than twofold up/down regulated probes and correction for multiple testing by the Benjamini-Hochberg method was applied. Probes with a fold change $>2$ and a false discovery rate of $<0.05$ in (A) ATP1A1/2B3 mutated tumors compared to KCNJ5 mutated tumors and (B) WT tumors compared to KCNJ5 mutated tumors, displayed in red.

Fernandes-Rosa et al. 2014). Conserved status throughout evolution and its location suggest a functional consequence on protein function. Compared to previously analyzed cohorts from Western countries, we observed relatively few CACNA1D mutants (Azizan et al. 2013, Scholl et al. 2013, Fernandes-Rosa et al. 2014). We re-sequenced all parts of CACNA1D previously reported as mutated in APAs. We also expanded our analysis to areas where missense mutations have resulted in a gain of function effect in other VDCCs (Ducros et al. 2001, Pietrobon 2002, Hoda et al. 2005, Striessnig et al. 2010, Stockner \& Koschak 2013). Interestingly, in two studies conducted in Asia, only two mutations in 282 analyzed APAs were found (Wang et al. 2015, Zheng et al. 2015). They also observed a high prevalence of KCNJ5 mutations, 76.2\% (215/282). Similar to the Asian cohorts, our prevalence of KCNJ5 mutations was relatively high. Compared to another large European cohort of 474 APAs (Fernandes-Rosa et al. 2014), our patients were more often female and had larger adenomas, a phenotype associated with KCNJ5 mutations (Akerstrom et al. 2012, Azizan et al. 2012a, Fernandes-Rosa et al. 2014). This could potentially explain our relatively low number of CACNA1D mutations, which seem to occur in smaller tumors in male patients (Azizan et al. 2013, Scholl et al. 2013, Fernandes-Rosa et al. 2014). This affirms the notion that aside from a possible demographic bias, the diagnostic workup and selection of patients affect the distribution of mutations.

We detected ATP1A1 mutations in $6.1 \%$ of tumors. ATP1A1 encodes the alpha-1 subunit of a ubiquitously expressed $\mathrm{Na}^{+} / \mathrm{K}^{+}$ATPase, vital for establishing the membrane potential in ZG cells (Spät \& Hunyady 2004). The mutations were either located close to or involving amino acid Leu104 in the M1 domain, or affecting the Glu960 and Glu961 residues of the M9 domain. Leu104 has been proposed to interact with Glu334 in the

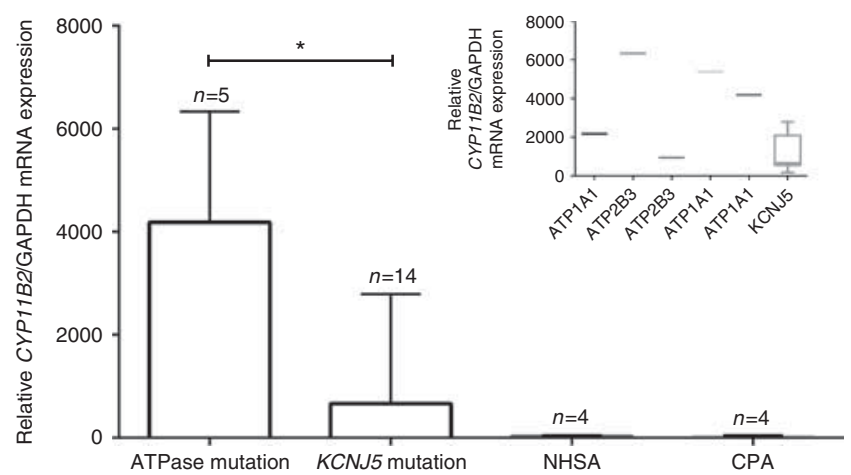

Figure 4

mRNA expression of CYP11B2 in ATP1A1/2B3 and KCNJ5 mutated adenomas. Relative mRNA expression $\left(2^{(-\Delta \Delta C T)}\right)$ of CYP11B2 in ATP1A1 and ATP2B3 mutated APAs = ATPase compared to KCNJ5 mutated, NHSA and CPA. GAPDH was used as an internal control. The relative level of CYP11B2 was compared between ATPase and KCNJ5 mutated tumors using a MannWhitney's $U$-test. Median and maximum values are displayed. NHSA, nonhormone secreting adenoma; CPA, cortisol producing adenoma; ${ }^{\star} P<0.05$. Insert depicts the individual levels of CYP11B2 in the ATP1A1/2B3 mutated tumors (median and range).

Published by Bioscientifica Ltd. 


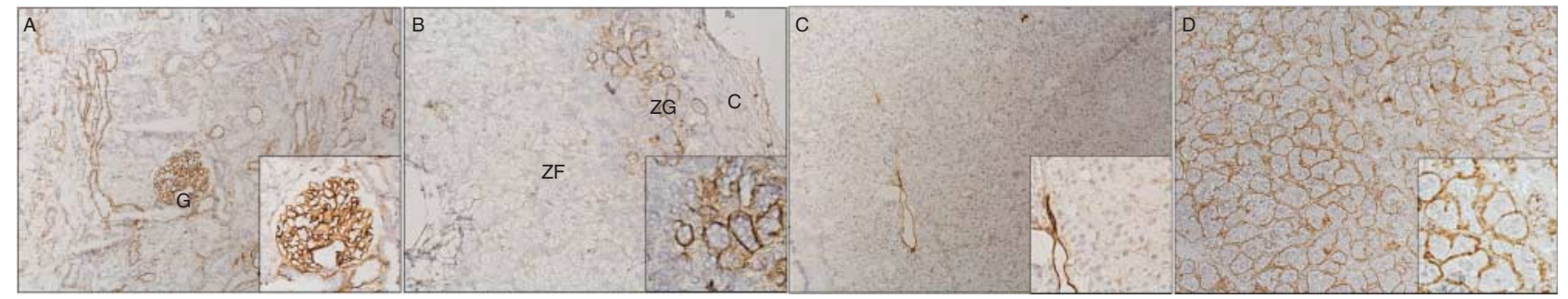

\section{Figure 5}

Immunohistochemistry for NPNT. Protein expression for NPNT by immunohistochemistry. (A) Normal kidney tissue. (B) Normal adrenal tissue adjacent to a non-hormone secreting adenoma. (C) Representative staining of $K C N J 5$ mutated tumor tissues. (D) Representative staining of ATP1A1/ATP2B3 mutated tissues. G, glomeruli; C, capsule; ZG, zona glomerulosa; ZF, zona fasciculata. transmembrane helix M4, leading to potassium binding and occlusion by the channel (Einholm et al. 2007). P.Leu104Arg and p.Gly99Arg mutations likely disrupt this site, and have been shown to increase depolarization, CYP11B2 expression and aldosterone production in vitro (Azizan et al. 2013, Williams et al. 2014). The mutations in the M9 domain were novel deletions affecting amino acids Glu960 and Glu961 in the third $\mathrm{Na}^{+}$binding site of the protein. Previous in vitro studies of a p.Glu961Ala mutant showed reduced $\mathrm{Na}^{+}$binding and a lowered affinity for $\mathrm{K}^{+}$(Li et al. 2005). Mutations in ATP1A1 have previously not been described in any syndromes, and no germline mutation could be detected in screened familial PA cases (Beuschlein et al. 2013). Given its ubiquitous expression and vital role for establishing the membrane potential, this is not surprising (Jorgensen et al. 2003). However, an association between ATP1A1 haplotypes and primary hypertension has been described (Herrera et al. 1998, Treva Rice et al. 2000, Glorioso et al. 2007). Further investigation of the functional consequences of ATP1A1 genotypes and haplotypes in hypertensive cohorts are therefore warranted.

ATP2B3 encodes a plasma membrane $\mathrm{Ca}^{2+}$ ATPase that actively transports $\mathrm{Ca}^{2+}$ out of the cell, resulting in low intracellular $\mathrm{Ca}^{2+}$ levels, thereby inhibiting aldosterone production (Spät \& Hunyady 2004). We observed five APAs with ATP2B3 mutations, including two novel deletions and two previously reported deletions (Beuschlein et al. 2013, Williams et al. 2014). The mutations span a seven amino acid region in the M4 domain, a part of the protein highly conserved among orthologous. Importantly, these amino acids are located at similar positions as the mutated residues in the M4 domain in ATP1A1. In contrast to $A T P 1 A 1$, a germline $A T P 2 B 3$ mutation has been found in a family with X-linked congenital cerebellar atrophy (Zannia et al. 2012). Functional studies showed that this mutation (p.Gly1107Asp) decreased the ability of the channel to extrude $\mathrm{Ca}^{2+}$ from the cells. To our knowledge, hyperaldosteronism has not been observed in these patients.

Previous studies have described genotype-phenotype correlations in APAs. While some have not described any gender association (Scholl et al. 2013, Williams et al. 2014), we could confirm that $A T P 1 A 1 / 2 B 3 / C A C N A 1 D$ mutated tumors are more often found in male patients, compared to a female overrepresentation for KCNJ5 mutated tumors (Azizan et al. 2013, Beuschlein et al. 2013, Dutta et al. 2013, Fernandes-Rosa et al. 2014). We also observed an older age at the time of operation for patients with these tumors, previously reported by some (Azizan et al. 2013, Scholl et al. 2013, Fernandes-Rosa et al. 2014) but not by others (Beuschlein et al. 2013, Dutta et al. 2013, Williams et al. 2014). We could not confirm higher preoperative aldosterone levels (Beuschlein et al. 2013), but observed the previously reported association with smaller tumors (Azizan et al. 2013, Dutta et al. 2013, Scholl et al. 2013, Fernandes-Rosa et al. 2014). Importantly, due to their smaller size $A T P 1 A 1 / 2 B 3 / C A C N A 1 D$ mutated tumors may be missed in patients that have not undergone AVS, which may also lead to delayed diagnosis and subsequently an older age at the time of the operation. It also suggests that the use of only CT/MRI may bias the mutational spectrum towards more KCNJ5 mutations, as previously indicated (Fernandes-Rosa et al. 2014).

Previous reports have described a ZG-like histological appearance in $A T P 1 A 1 / 2 B 3 / C A C N A 1 D$ mutated tumors compared to a more ZF-like composition in KCNJ5 mutated tumors (Azizan et al. 2013, Dekkers et al. 2014, Monticone et al. 2015). Also, high CYP11B2 and low CYP11B1 protein expression have been observed in these APAs (Azizan et al. 2013, Dekkers et al. 2014, Monticone et al. 2015). However, a large transcriptomic analysis of

Published by Bioscientifica Ltd. 


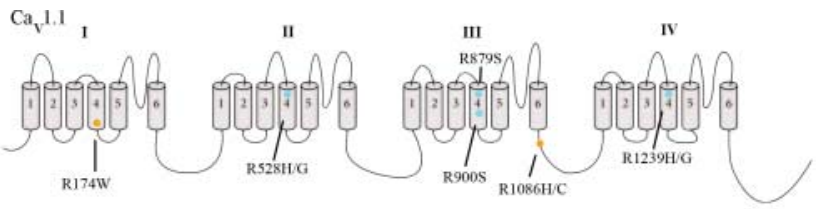

$\mathrm{Ca}_{\mathrm{V}^{1.2}}{ }_{\mathrm{I}} \mathrm{II}$ III
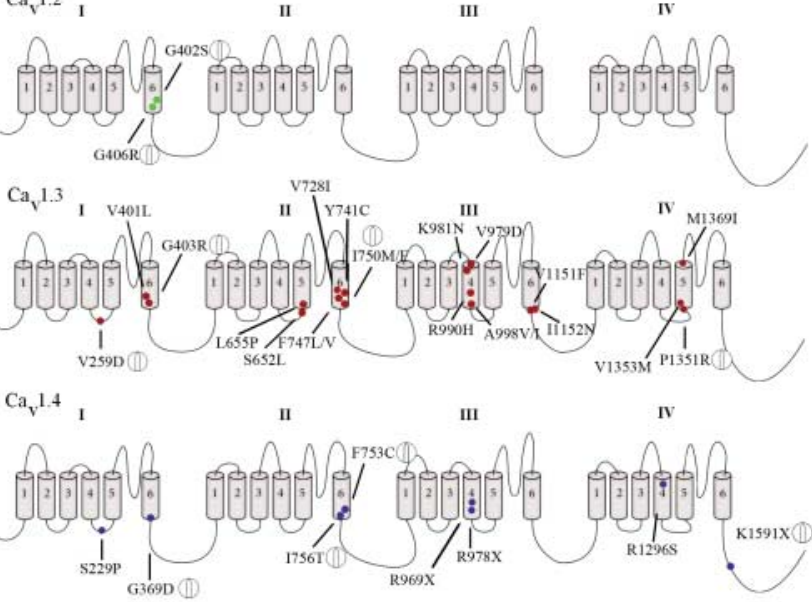

$\mathrm{Ca}_{\mathrm{v}} 2.1$

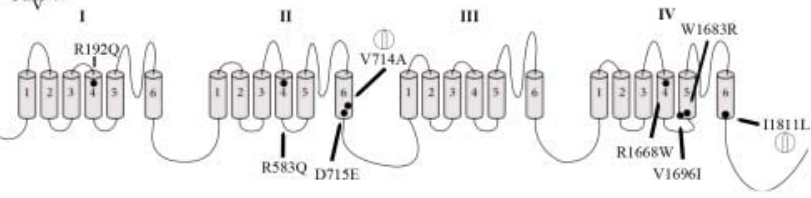

Figure 6

Illustration depicting mutations in different voltage-dependent calcium channels $\alpha 1$-subunits. Color indicates different disorders associated with each isoform and mutation ( $\mathrm{Ca}_{\mathrm{v}} 1.3$ is the product of CACNA1D). V728I, F747V, I750M and V1353M correspond to V748I, F767V, I770M and V1373M (NP_001122312, NP_000711). P1351R, V1353M and M1369l correspond to P1336R, V1338M and M1354I (NP_001122312, NP_001122311). Orange, malignant hyperthermia susceptibility; Light blue, hypokalemic periodic paralysis type 1; Green, timothy syndrome; Red, primary aldosteronism; Dark blue, congenital stationary blindness; Black dots, familial hemiplegic migraine; A circle with an arrow indicates a gain of function mutation shown by in vitro studies (Pietrobon 2002, Striessnig et al. 2010, Azizan et al. 2013, Scholl et al. 2013, Stockner \& Koschak 2013, Fernandes-Rosa et al. 2014, Wang et al. 2015).

92 APAs did not detect any differences in CYP11B2 and CYP11B1 expression (Fernandes-Rosa et al. 2014). Neither did it detect a specific transcriptome signature (Boulkroun et al. 2012) nor a specific histological appearance (Fernandes-Rosa et al. 2014). Our transcriptomic analysis revealed differences between KCNJ5 mutated APAs and the other tumors. However, because of the low number of samples, our results should be interpreted with some consideration. Interestingly, of the two transcripts displaying higher expression in our ATP1A1/2B3 mutated tumors, NPNT was previously described as a ZG marker and the second most upregulated transcript in
ATP1A1/CACNA1D mutated tumors compared to KCNJ5 mutated tumors (Azizan et al. 2013). NPNT encodes nephronectin, an extracellular matrix protein important for kidney development (Linton et al. 2007). Immunohistochemistry verified nephronectin as a possible ZG cell marker and confirmed higher expression in ATP1A1/2B3 mutated tumors compared to KCNJ5 mutated tumors. We also confirmed previous reports of higher CYP11B2 expression in our $A T P 1 A 1 / 2 B 3$ mutated tumors. The lower CYP11B2 expression in APAs harboring KCNJ5 mutations may indicate a relatively lower production of aldosterone per cell, possibly compensated by their larger size, explaining the similar serum levels of aldosterone in patients with $A T P 1 A 1 / 2 B 3 / C A C N A 1 D$ mutated tumors. Our results provide additional evidence that $K C N J 5$ mutated APAs do display subtle differences in their molecular phenotype compared to ATP1A1/2B3 mutated tumors (Azizan et al. 2012b, Azizan et al. 2013, Dekkers et al. 2014, Monticone et al. 2015). Perhaps these small variances reflect a different cellular origin of KCNJ5 mutated APAs, but because of similar intracellular events (i.e., increased $\mathrm{Ca}^{2+}$ ) these remain subtle. Of note, most KCNJ5 mutated APAs express markers of ZG cells such as DAB2 (Boulkroun et al. 2010), making the notion of different cellular origins more complex.

A limitation of this study was that we re-sequenced selected regions of these genes, possibly lowering the true prevalence of mutations. The entire coding segments of ATP1A1 and ATP2B3 have previously been analyzed in a large cohort of APAs (Beuschlein et al. 2013), and the results suggest that mutations outside these areas are rare. Mutations in CACNA1D have been found in hotspot areas in multiple exons. We chose to sequence all areas in CACNA1D where mutations previously have been described in APAs, and also included areas where gain of function mutations have been observed in other disorders. We could not detect any mutations outside mutational hotspot areas. If a gain of function effect is the cause of these tumors, the chance of finding other prevalent mutational areas seems less likely but cannot be excluded. Also, we did not perform functional characterization of our novel mutations; however, due to the conservation in different organisms and their location, we infer an altered protein function.

In conclusion, somatic and germline mutations in regulators of the membrane potential and/or intracellular $\mathrm{Ca}^{2+}$ homeostasis occur frequently in APAs. Also, non mutated tumors display a similar mRNA signature as $A T P 1 A 1 / 2 B 3$ mutated tumors. This makes it tempting to speculate that additional proteins involved in this

Published by Bioscientifica Ltd 
regulatory pathway are important for the tumorigenesis. With improved understanding of these events, the possibility of more specific treatment and better diagnostics will increase.

\section{Supplementary data}

This is linked to the online version of the paper at http://dx.doi.org/10.1530/ ERC-15-0321.

\section{Declaration of interests}

The authors declare that there is no conflict of interest that could be perceived as prejudicing the impartiality of the research reported.

\section{Funding}

This work was supported by grants from the Swedish Cancer Society, Swedish Research Council, Selander Foundation and the LIONS cancer research fund.

\section{Author contribution statement}

Author contributions: T Åkerström performed experiments, carried out statistical analysis and drafted the manuscript. T A kerström, S Backman, H S Willenberg, K Cupisti, J Ip, A Moser, R Maharjan, B Robinson, K A Iwen, H Dralle, C D Volpe, M Bäckdahl, J Botling, P Stålberg, G Westin, M K Walz, H Lehnert, S Sidhu, J Zedenius, P Björklund and P Hellman participated in either the acquisition, analysis, or interpretation of data. All authors contributed with critical revision of the manuscript and all authors performed administrative, technical or material support. P Bjorklund and $P$ Hellman obtained funding. T Åkerström and P Björklund designed the study. P Björklund supervised the study.

\section{Acknowledgements}

We would like to thank Birgitta Bondeson for all her help and guidance at the laboratory, and Joakim Crona for critically reviewing the manuscript. We would also like to extend our gratitude to LIONS cancer research fund for generously contributing to our research. Finally, we would like to thank all the patients, without whom we would not have been able to conduct this study.

\section{References}

Akerstrom T, Crona J, Delgado Verdugo A, Starker LF, Cupisti K, Willenberg HS, Knoefel WT, Saeger W, Feller A, Ip J et al. 2012 Comprehensive re-sequencing of adrenal aldosterone producing lesions reveal three somatic mutations near the KCNJ5 potassium channel selectivity filter. PLOS ONE 7 e41926. (doi:10.1371/journal. pone.0041926)

Azizan EA, Murthy M, Stowasser M, Gordon R, Kowalski B, Xu S, Brown MJ \& O'Shaughnessy KM 2012a Somatic mutations affecting the selectivity filter of KCNJ5 are frequent in 2 large unselected collections of adrenal aldosteronomas. Hypertension 59 587-591. (doi:10.1161/HYPERTENSIONAHA.111.186239)

Azizan EA, Lam BY, Newhouse SJ, Zhou J, Kuc RE, Clarke J, Happerfield L, Marker A, Hoffman GJ \& Brown MJ 2012b Microarray, qPCR, and KCNJ5 sequencing of APAs reveal differences in genotype and phenotype between zona glomerulosa- and zona fasciculata-like tumors. Journal of Clinical Endocrinology and Metabolism 97 E819-E829. (doi:10.1210/jc.2011-2965)

Azizan EA, Poulsen H, Tuluc P, Zhou J, Clausen MV, Lieb A, Maniero C, Garg S, Bochukova EG, Zhao W et al. 2013 Somatic mutations in ATP1A1 and CACNA1D underlie a common subtype of adrenal hypertension. Nature Genetics 45 1055-1060. (doi:10.1038/ng.2716)

Beuschlein F, Boulkroun S, Osswald A, Wieland T, Nielsen HN, Lichtenauer UD, Penton D, Schack VR, Amar L, Fischer E et al. 2013 Somatic mutations in ATP1A1 and ATP2B3 lead to aldosteroneproducing adenomas and secondary hypertension. Nature Genetics $\mathbf{4 5}$ 440-444. (doi:10.1038/ng.2550)

Boulkroun S, Samson-Couterie B, Dzib JF, Lefebvre H, Louiset E, Amar L, Plouin PF, Lalli E, Jeunemaitre X, Benecke A et al. 2010 Adrenal cortex remodeling and functional zona glomerulosa hyperplasia in primary aldosteronism. Hypertension 56 885-892. (doi:10.1161/HYPERTENSIONAHA.110.158543)

Boulkroun S, Beuschlein F, Rossi GP, Golib-Dzib JF, Fischer E, Amar L, Mulatero P, Samson-Couterie B, Hahner S, Quinkler M et al. 2012 Prevalence, clinical, and molecular correlates of KCNJ5 mutations in primary aldosteronism. Hypertension 59 592-598. (doi:10.1161/ HYPERTENSIONAHA.111.186478)

Brilla CG \& Weber K 1992 Mineralocorticoid excess, dietary sodium, and myocardial fibrosis. Journal of Laboratory and Clinical Medicine 120 893-901.

Catterall W 2010 Signaling complexes of voltage-gated sodium and calcium channels. Neuroscience Letters 486 107-116. (doi:10.1016/ j.neulet.2010.08.085)

Choi M, Scholl UI, Yue P, Bjorklund P, Zhao B, Nelson-Williams C, Ji W, Cho Y, Patel A, Men CJ et al. $2011 \mathrm{~K}^{+}$channel mutations in adrenal aldosterone-producing adenomas and hereditary hypertension. Science 331 768-772. (doi:10.1126/science.1198785)

Cohen CJ, McCarthy RT, Barrett PQ \& Rasmussen H 1988 Ca channels in adrenal glomerulosa cells: $\mathrm{K}^{+}$and angiotensin II increase T-type Ca channel current. PNAS 85 2412-2416. (doi:10.1073/pnas.85.7.2412)

Dekkers T, ter Meer M, Lenders JW, Hermus AR, Schultze Kool L, Langenhuijsen JF, Nishimoto K, Ogishima T, Mukai K, Azizan EA et al. 2014 Adrenal nodularity and somatic mutations in primary aldosteronism: one node is the culprit? Journal of Clinical Endocrinology and Metabolism 99 E1341-E1351. (doi:10.1210/jc.2013-4255)

Ducros A, Denier C, Joutel A, Cecillon M, Lescoat C, Vahedi K, Darcel F, Vicaut E, Bousser MG \& Tournier-Lasserve E 2001 The clinical spectrum of familial hemiplegic migraine associated with mutations in a neuronal calcium channel. New England Journal of Medicine 345 17-24. (doi:10.1056/NEJM200107053450103)

Dutta RK, Welander J, Brauckhoff M, Walz M, Alesina P, Arnesen T, Söderkvist P \& Gimm O 2013 Complementary somatic mutations of KCNJ5, ATP1A1 and ATP2B3 in sporadic aldosterone producing adrenal adenomas. Endocrine-Related Cancer 21 L1-L4. (doi:10.1530/ ERC-13-0466)

Einholm AP, Andersen JP \& Vilsen B 2007 Importance of Leu99 in transmembrane segment $\mathrm{M} 1$ of the $\mathrm{Na}^{+}, \mathrm{K}^{+}$- ATPase in the binding and occlusion of $\mathrm{K}^{+}$. Journal of Biological Chemistry 282 23854-23866. (doi:10.1074/jbc.M702259200)

Fallo F, Pezzi V, Barzon L, Mulatero P, Veglio F, Sonino N \& Mathis JM 2012 Quantitative assessment of CYP11B1 and CYP11B2 expression in aldosterone-producing adenomas. European Journal of Endocrinology 147 795-802. (doi:10.1530/eje.0.1470795)

Fernandes-Rosa FL, Williams TA, Riester A, Steichen O, Beuschlein F, Boulkroun S, Strom TM, Monticone S, Amar L, Meatchi T et al. 2014 Genetic spectrum and clinical correlates of somatic mutations in aldosterone-producing adenoma. Hypertension 64 354-361. (doi:10.1161/HYPERTENSIONAHA.114.03419)

Fogari R, Preti P, Zoppi A, Rinaldi A, Fogari E \& Mugellini A 2007 Prevalence of primary aldosteronism among unselected hypertensive patients: a prospective study based on the use of an aldosterone/renin ratio 
above 25 as a screening test. Hypertension Research 30 111-117. (doi:10.1291/hypres.30.111)

Glorioso N, Herrera VL, Bagamasbad P, Filigheddu F, Troffa C, Argiolas G, Bulla E, Decano JL \& Ruiz-Opazo N 2007 Association of ATP1A1 and dear single-nucleotide polymorphism haplotypes with essential hypertension: sex-specific and haplotype-specific effects. Circulation Research 100 1522-1529. (doi:10.1161/01.RES.0000267716. 96196.60)

Hannemann A \& Wallaschofski H 2012 Prevalence of primary aldosteronism in patient's cohorts and in population-based studies - a review of the current literature. Hormone and Metabolic Research 44 157-162. (doi:10.1055/s-0031-1295438)

Herrera VL, Xie HX, Lopez LV, Schork NJ \& Ruiz-Opazo N 1998 The $\alpha 1$ $\mathrm{Na}, \mathrm{K}-\mathrm{ATPa}$ e gene is a susceptibility hypertension gene in the Dahl saltsensitiveHSD rat. Journal of Clinical Investigation 102 1102-1111. (doi:10.1172/JCI3868)

Hoda JC, Zaghetto F, Koschak A \& Striessnig J 2005 Congenital stationary night blindness type 2 mutations S229P, G369D, L1068P, and W1440X alter channel gating or functional expression of Cav1.4 L-type $\mathrm{Ca}^{2+}$ channels. Journal of Neuroscience 25 252-259. (doi:10.1523/JNEUROSCI.3054-04.2005)

Jorgensen PL, Håkansson KO \& Karlish SJ 2003 Structure and mechanism of Na,K-ATPase: functional sites and their interactions. Annual Review of Physiology 65 817-849. (doi:10.1146/annurev.physiol.65.092101. 142558)

Kitamoto T, Suematsu S, Matsuzawa Y, Saito J, Omura M \& Nishikawa T 2015 Comparison of cardiovascular complications in patients with and without KCNJ5 gene mutations harboring aldosterone-producing adenomas. Journal of Atherosclerosis and Thrombosis 22 191-200. (doi:10.5551/jat.24455)

Larkin MA, Blackshields G, Brown NP, Chenna R, McGettigan PA, McWilliam H, Valentin F, Wallace IM, Wilm A, Lopez R et al. 2007 Clustal W and Clustal X version 2.0. Bioinformatics 23 2947-2948. (doi:10.1093/bioinformatics/btm404)

Lenzini L, Rossitto G, Maiolino G, Letizia C, Funder JW \& Rossi GP 2015 A meta-analysis of somatic KCNJ5 $\mathrm{K}^{+}$channel mutations in 1636 patients with an aldosterone-producing adenoma. Journal of Clinical Endocrinology and Metabolism [in press]. (doi:10.1210/jc.2015-214)

Li C, Capendeguy O, Geering K \& Horisberger JD 2005 A third $\mathrm{Na}^{+}$-binding site in the sodium pump. PNAS 102 12706-12711. (doi:10.1073/pnas. 0505980102)

Linton JM, Martin GR \& Reichardt LF 2007 The ECM protein nephronectin promotes kidney development via integrin $\alpha 8 \beta 1$-mediated stimulation of Gdnf expression. Development 134 2501-2509. (doi:10.1242/dev. 005033)

Livak KJ \& Schmittgen TD 2001 Analysis of relative gene expression data using real-time quantitative PCR and the $2(-\Delta \Delta \mathrm{C}(\mathrm{T}))$ method. Methods 25 402-408. (doi:10.1006/meth.2001.1262)

Monticone S, Castellano I, Versace K, Lucatello B, Veglio F, Gomez-Sanchez CE, Williams TA \& Mulatero P 2015 Immunohistochemical, genetic and clinical characterization of sporadic aldosterone-producing adenomas. Molecular and Cellular Endocrinology 411 146-154. (doi:10.1016/j.mce.2015.04.022)

Oki K, Plonczynski MW, Luis Lam M, Gomez-Sanchez EP \& Gomez-Sanchez CE 2012 Potassium channel mutant KCNJ5 T158A expression in HAC-15 cells increases aldosterone synthesis. Endocrinology 153 1774-1782. (doi:10.1210/en.2011-1733)

Pietrobon D 2002 Calcium channels and channelopathies of the central nervous system. Molecular Neurobiology 25 31-50. (doi:10.1385/ $\mathrm{MN}: 25: 1: 031$
Quinn SJ, Cornwall MC \& Williams GH 1987 Electrical properties of isolated rat adrenal glomerulosa and fasciculata cells. Endocrinology $\mathbf{1 2 0}$ 903-914. (doi:10.1210/endo-120-3-903)

Rice T, Rankinen T, Province MA, Chagnon YC, Pérusse L, Borecki IB, Bouchard C \& Rao DC 2000 Genome-wide linkage analysis of systolic and diastolic blood pressure: the Québec family study. Circulation 102 1956-1963. (doi:10.1161/01.CIR.102.16.1956)

Rossi GP, Sacchetto A, Pavan E, Palatini P, Graniero GR, Canali C \& Pessina AC 1997 Remodeling of the left ventricle in primary aldosteronism due to Conn's adenoma. Circulation 95 1471-1478. (doi:10.1161/01.CIR.95.6.1471)

Rossi GP, Bernini G, Caliumi C, Desideri G, Fabris B, Ferri C, Ganzaroli C, Giacchetti G, Letizia C, Maccario M et al. 2006 A prospective study of the prevalence of primary aldosteronism in 1,125 hypertensive patients. Journal of the American College of Cardiology 48 2293-2300. (doi:10.1016/j.jacc.2006.07.059)

Savard S, Amar L, Plouin PF \& Steichen O 2013 Cardiovascular complications associated with primary aldosteronism: a controlled cross-sectional study. Hypertension 62 331-336. (doi:10.1161/HYPERTENSIONAHA.113.01060)

Scholl UI, Goh G, Stolting G, de Oliveira RC, Choi M, Overton JD, Fonseca AL, Korah R, Starker LF, Kunstman JW et al. 2013 Somatic and germline CACNA1D calcium channel mutations in aldosteroneproducing adenomas and primary aldosteronism. Nature Genetics $\mathbf{4 5}$ 1050-1054. (doi:10.1038/ng.2695)

Scholl UI, Stölting G, Nelson-Williams C, Vichot AA, Choi MLE, Prasad ML, Goh G, Carling T, Juhlin CC, Quack I et al. 2015 Recurrent gain of function mutation in calcium channel CACNA1H causes early-onset hypertension with primary aldosteronism. eLife 4 e06315. (doi:10.7554/eLife.06315)

Spät A \& Hunyady L 2004 Control of aldosterone secretion: a model for convergence in cellular signaling pathways. Physiological Reviews $\mathbf{8 4}$ 489-539. (doi:10.1152/physrev.00030.2003)

Stockner T \& Koschak A 2013 What can naturally occurring mutations tell us about $\mathrm{Ca}(\mathrm{v}) 1$.x channel function? Biochimica et Biophysica Acta $\mathbf{1 8 2 8}$ 1598-1607. (doi:10.1016/j.bbamem.2012.11.026)

Striessnig J, Bolz HJ \& Koschak A 2010 Channelopathies in Cav1.1, Cav1.3, and Cav1.4 voltage-gated L-type $\mathrm{Ca}^{2+}$ channels. Pflügers Archiv 460 361-374. (doi:10.1007/s00424-010-0800-x)

Taguchi R, Yamada M, Nakajima Y, Satoh T, Hashimoto K, Shibusawa N, Ozawa A, Okada S, Rokutanda N, Takata D et al. 2012 Expression and mutations of KCNJ5 mRNA in Japanese patients with aldosteroneproducing adenomas. Journal of Clinical Endocrinology and Metabolism 97 1311-1319. (doi:10.1210/jc.2011-2885)

Wang B, Li X, Zhang X, Ma X, Chen L, Zhang Y, Lyu X, Tang Y, Huang Q, Gao $Y$ et al. 2015 Prevalence and characterization of somatic mutations in chinese aldosterone-producing adenoma patients. Medicine 94 e708. (doi:10.1097/MD.0000000000000708)

Williams TA, Monticone S, Schack VR, Stindl J, Burrello J, Buffolo F, Annaratone L, Castellano I, Beuschlein F, Reincke M et al. 2014 Somatic ATP1A1, ATP2B3, and KCNJ5 mutations in aldosterone-producing adenomas. Hypertension 63 188-195. (doi:10.1161/HYPERTENSIONAHA.113.01733)

Zannia G, Calìb T, Kalscheuerc VM, Ottolinid D, Barresia S, Lebrune N, Montecchi-Palazzif L, Huc H, Chellye J, Bertinia E et al. 2012 Mutation of plasma membrane $\mathrm{Ca}^{2+}$ ATPase isoform 3 in a family with X-linked congenital cerebellar ataxia impairs $\mathrm{Ca}^{2+}$ homeostasis. PNAS 109 14514-14519. (doi:10.1073/pnas.1207488109)

Zheng FF, Zhu LM, Nie AF, Li XY, Lin JR, Zhang K, Chen J, Zhou WL, Shen ZJ, Zhu YC et al. 2015 Clinical characteristics of somatic mutations in chinese patients with aldosterone-producing adenoma. Hypertension 65 622-628. (doi:10.1161/HYPERTENSIONAHA.114.03346)

Received in final form 20 July 2015

Accepted 21 July 2015 http://erc.endocrinology-journals.org DOI: $10.1530 /$ ERC-15-0321
(C) 2015 Society for Endocrinology Printed in Great Britain
Published by Bioscientifica Ltd 